\title{
Constraining the Si composition and thermal history of Earth's liquid core from ab initio calculations.
}

\author{
Alfred J. Wilson \\ School of Earth and Environment, University of Leeds \\ a.j.wilson1@leeds.ac.uk
}

Monica Pozzo

Department of Earth Sciences, University College London

London Centre for Nanotechnology, Thomas Young Centre, University College London m.pozzo@ucl.ac.uk

Dario Alfé

Department of Earth Sciences, University College London

London Centre for Nanotechnology, Thomas Young Centre, University College London Dipartimento di Fisica "Ettore Pancini”, Universita' di Napoli

d.alfe@ucl.ac.uk

Andrew M. Walker

Department of Earth Sciences, University of Oxford andrew.walker@earth.ox.ac.uk

Sam Greenwood

School of Earth and Environment, University of Leeds

S.Greenwood@leeds.ac.uk

Anne Pommier

Earth \& Planets Laboratory, Carnegie Institution for Science

apommier@carnegiescience.edu

Christopher J. Davies

School of Earth and Environment, University of Leeds

c.davies@leeds.ac.uk

This is a non-peer reviewed, pre-print manuscript submitted to EarthArXiv, also submitted to Earth and Planetary Science Letters. 
1 Highlights

2 Constraining the Si composition and thermal history of Earth's 3 liquid core from ab initio calculations.

4 Alfred J. Wilson, Monica Pozzo, Dario Alfè, Andrew M. Walker, Sam Green-

5 wood, Anne Pommier, Christopher J. Davies

6

7 liquids at core conditions provide equilibrium constant for silicon be$8 \quad$ tween core and mantle.

9

10

- First ab initio results of silicon partitioning between silicate and iron

- A thermodynamic model fit to previous experiments and confirmed by our ab initio calculations describes partitioning of silicon and oxygen between core and mantle, placing bounds on modern Si content of the core at $\sim 1.8-4.5 \mathrm{wt} \%$.

- Silicon precipitation rates are lower than previous studies but still provide ample power to sustain the ancient magnetic field.

- Coupled models of core-mantle evolution with Si precipitation satisfying observational constraints predict an inner core age of 840-940 Ma and a long lived basal magma ocean. 


\title{
Constraining the Si composition and thermal history of Earth's liquid core from ab initio calculations.
}

\author{
Alfred J. Wilson ${ }^{\mathrm{a}, *}$, Monica Pozzo $^{\mathrm{b}, \mathrm{c}}$, Dario Alfè ${ }^{\mathrm{b}, \mathrm{c}, \mathrm{d}}$, Andrew M. Walker ${ }^{\mathrm{e}}$, \\ Sam Greenwood $^{\mathrm{a}}$, Anne Pommier ${ }^{\mathrm{f}}$, Christopher J. Davies ${ }^{\mathrm{a}}$ \\ ${ }^{a}$ School of Earth and Environment, University of Leeds, Woodhouse, Leeds, LS2 \\ 9JT, UK \\ ${ }^{b}$ Department of Earth Sciences, University College London, 5 Gower \\ Place, London, WC1E 6BS, UK \\ ${ }^{c}$ London Centre for Nanotechnology, Thomas Young Centre, University College \\ London, 17-19 Gordon Street, London, WC1H 0AH, UK \\ ${ }^{d}$ Dipartimento di Fisica "Ettore Pancini", Universita" di Napoli "Federico II", Monte S. \\ Angelo, Napoli, 80126, Italy \\ ${ }^{e}$ Department of Earth Sciences, University of Oxford, S Parks Rd, Oxford, OX1 \\ $3 A N, U K$ \\ ${ }^{f}$ Earth 83 Planets Laboratory, Carnegie Institution for Science, 5241 Broad Branch \\ Road, Washington DC, 20015, USA
}

\section{Abstract \\ Earth's core has sustained a global magnetic field for much of the last 4 bil- lion years which is, at present, sustained by the power associated with inner core growth. High thermal conductivity of the core suggests the solid inner core is young and models of this predict that there is insufficient power from secular cooling to sustain a geodynamo prior to inner core formation. Pre- cipitation of light elements dissolved into the liquid core offers an alternative power source for the magnetic field in the absence of inner core growth. We present the first ab initio calculations of the silicon partition coefficient at core-mantle boundary conditions and a thermodynamic partitioning model based on interaction parameters which captures previous experimental re-}


sults. We report our model and its implications for the past and present core composition as well as the effect of silicon precipitation on the early geodynamo. Oxygen competes with silicon in the liquid metal, meaning for one to be abundant, the other must be sparse. We calculate precipitation rates of $\sim 10^{-4}$ to $10^{-6}$ wt $\% \mathrm{~K}^{-1}$ for oxygen concentrations of 0.6 to 3.1 wt\%. Incorporating our partitioning model into a classic thermal evolution model of the core coupled to a parameterised model of the solid mantle, we show that precipitation of Si can satisfy constraints of the present inner core size, convective heat flux of the mantle and mantle temperature, all whilst sustaining a magnetic field until inner core formation, but requires that the initial oxygen content of the core was $<3 \mathrm{wt} \%$. We find that the core inner age is between 840 and 940 Myrs and that the ancient core was hot, with a core mantle boundary temperature of $\sim 4700 \mathrm{~K}, 3.5$ Ga.

Keywords: Ab Initio, Earth's Core Chemistry, Thermal Evolution, Silicon PACS: 0000, 1111

2000 MSC: 0000, 1111

\section{Introduction}

Earth's magnetic field is important for the habitability of our planet and yet the power to sustain it remains enigmatic for the majority of geological time. Palaeointensity data suggest the field has been maintained for at least the last 3.45 Gyrs (Tarduno et al., 2010) but the main power sources derive from growth of the inner core, which probably started in the last $\sim 1$ 
Gyrs (Labrosse et al., 2001; Nimmo, 2015a; Davies et al., 2019). Growth of the inner core provides latent heat, however more influential is that light elements can be partitioned to the liquid (Braginsky, 1963) creating a chemical buoyancy source at the base of the outer core. Oxygen is considered a likely candidate here as it can help to explain the density contrast between the inner and outer core (Alfè et al., 2002) although similar partitioning and convective influence can be explained by carbon (Li et al., 2019). Enrichment of light elements provides power for outer core convection which is expected to be the major contributor to geodynamo power today (Buffett et al., 1996; Gubbins et al., 2004; Labrosse, 2015).

To power the geodynamo before inner core growth, rapid cooling rates are needed. Several first principles calculations and high pressure $(\mathrm{P})$ and temperature $(\mathrm{T})$ experimental results suggest that the core thermal conductivity may be significantly higher than previously thought (Pozzo et al., 2012; de Koker et al., 2012; Gomi et al., 2013; Zhang et al., 2020, 2022). Maintaining sufficient power for the dynamo with high conductivity constrains the inner core to be far less than 1 Gyrs old and requires the geodynamo to be powered by heat loss from the core alone. This rapidly cooling scenario means the mantle would have been subject to a super-solidus core-mantle boundary (CMB) temperature for much of Earth history (Nimmo, 2015b; Davies, 2015; Labrosse, 2015). Davies and Greenwood (2022) proposed that the presence of a basal magma ocean (BMO) may provide a resolution, although this approach relies upon the uncertain evolution of the BMO as well 
as requiring a conductivity at the lower limit of the recent high estimates.

In search of an alternate explanation for the long-lived geodynamo, prior studies have investigated whether light elements, incorporated during a hot differentiation, might become insoluble during cooling and precipitate from the liquid core. This precipitation would produce a positively buoyant precipitate and a residual, iron-rich, dense liquid which drives convection. Lower initial core temperatures and slower cooling allowed by this power source imply inner core ages closer to 1 Gyrs. $\mathrm{MgO}$ precipitation has been suggested (O'Rourke and Stevenson, 2016; Badro et al., 2016, 2018) although the dependence of magnesium solubility on the oxygen content of the core has been argued to reduce the overall power output, making $\mathrm{MgO}$ an insufficient power source for the geodynamo alone (Du et al., 2017, 2019). Others have investigated the possibility of $\mathrm{SiO}_{2}$ (Hirose et al., 2017; Helffrich et al., 2020) as well as simultaneous precipitation of multiple elements (Mittal et al., 2020). The predicted onset time and power generated by precipitation depend strongly on the thermodynamic model used to represent partitioning data. Badro et al. (2016) describe partitioning of Mg as only temperature and pressure dependent. Du et al. (2017) produce a composition dependent model but without interaction between chemical species. O'Rourke and Stevenson (2016), Hirose et al. (2017), Badro et al. (2018) and Helffrich et al. (2020) all implement an interaction parameter model (Ma, 2001) with differing numbers of included elements and interactions. Davies and Greenwood (2022) show that these differences change the onset time and power of precipitation 
significantly. Badro et al. (2018) apply the most rigorous thermodynamic model of these studies and lay a foundation for the analysis in this study.

The Mg content of the core is uncertain and high temperature differentiation of the core is required to incorporate sufficient $\mathrm{Mg}$ for later precipitation (O'Rourke and Stevenson, 2016; Badro et al., 2016). Here we examine Si partitioning. Si is a more widely accepted component in the liquid core (e.g. Takafuji et al. (2005); Rubie et al. (2015); Fischer et al. (2015)) and O is also expected to be dissolved into the core (Davies et al., 2020) making $\mathrm{SiO}_{2}$ the appropriate reactant in the silicate liquid. Additionally, the favourable solubility of $\mathrm{Si}$ in the core over $\mathrm{Mg}$ means that a cooler core formation is possible whilst still producing precipitation later (Hirose et al., 2017).

We produce new ab initio determinations of $\mathrm{SiO}_{2}$ partitioning at $\mathrm{CMB}$ conditions and show that these are in good agreement with previous experimental results. We derive a thermodynamic model for $\mathrm{SiO}_{2}$ partitioning using a dataset which spans a wide range of physical and chemical conditions than was available to previous studies and confirm this model with ab initio calculation. We use our model to describe partitioning in thermal history models of the cooling core. Hirose et al. (2017) evaluated the cooling rate needed to sustain a geodynamo from their experimentally derived precipitation rate and Mittal et al. (2020) implement a thermodynamic model of simultaneous precipitation of multiple light elements into a parameterised model of core thermal evolution. We take a similar approach, using a thermal evolution model whilst choosing to focus solely on Si precipitation with 
a greater number of resolved interaction parameters (including $\mathrm{C}, \mathrm{O}, \mathrm{Si}, \mathrm{S}$, $\mathrm{Mg}$ ) instead of several oxides with reduced compositional sensitivity.

\section{Methods}

To evaluate the influence of Si precipitation on the geodynamo we use ab initio molecular dynamic simulations of iron-rich liquids and silicate liquids to calculate equilibrium constants at CMB conditions. We compare our ab initio results to the results of a thermodynamic model fit to experimental partitioning data of Si between silicate and metallic liquids using the interaction parameter formulation of Ma (2001). This thermodynamic model is then coupled to a core evolution model to describe Si solubility in the liquid core as it cools. We then evaluate the influence of precipitation on the thermal history of the core.

\subsection{Ab Initio Calculations}

We conduct density functional theory (Hohenberg and Kohn, 1964; Kohn and Sham, 1965) molecular dynamic simulations of silicate and iron-rich liquids to calculate the excess chemical potentials of individual chemical components. Chemical potentials $\left(\mu_{i}\right)$ can be described as the free-energy change $(\partial F)$ of a system when the quantity of a species is changed

$$
\mu_{i}\left(v, T, x_{i}, x_{j}, \ldots\right)=\left(\frac{\partial F}{\partial x_{i}}\right)_{V, T, x_{i}, x_{j}, . .}
$$


in this case under conditions of constant volume ( $V, v$ is volume per atom), temperature $(T)$ and composition (where $x_{i}$ is the molar fraction of species $i$ ). Helmholtz free energy $(F)$ is used to match the constant volume conditions of our simulations. We use two complementary methods described in detail by Pozzo et al. (2019) to calculate $\mu_{i}$. Method 1 compares the $F$ of a reference system against another with a different number of solute atoms $\left(d N_{i}\right)$ to isolate $\mu_{i}$ of the solute:

$$
\mu_{i}\left(v, T, x_{i}, x_{j} \ldots\right)=\frac{F\left(V, T, x_{i}, x_{j}, \ldots\right)-F\left(V, T, x_{i}-d N_{i}, x_{j}, \ldots\right)}{d N} .
$$

Method 2 computes the change in free energy as a result of changing the number of solute atoms in the same system and calculating the change in free energy. The difference here is that the explicit free energies of two systems are not needed, only the change in free energy (for complete details, see Pozzo et al. (2019)).

To independently validate our thermodynamic model we find distribution coefficients $\left(K_{d}\right)$ from our ab initio results of $\mu_{i} u$. When $\mu_{i}$ on either side of a reaction are equal, this component is in thermodynamic equilibrium and each concentration will be stable

$$
\mu_{\mathrm{SiO}_{2}}^{\text {silicate }}\left(v, T, x_{\mathrm{SiO}_{2}}^{\text {silicate }}, x_{j}^{\text {silicate }}, \ldots\right)=\mu_{\mathrm{SiO}_{2}}^{\text {metal }}\left(v, T, x_{\mathrm{SiO}_{2}}^{\text {metal }}, x_{j}^{\text {metal }}, \ldots\right)
$$

Here $\mu_{i}$ is dependent on $v, T$ and composition. Separating out the configu- 
rational portion of $\mu_{i}$, which plays no role in partitioning, gives

$2\left(k_{B} T \ln x_{O}^{\text {silicate }}+\tilde{\mu}_{O}^{\text {silicate }}\right)+K_{B} T \ln x_{O}^{\text {silicate }}=2\left(k_{B} T \ln x_{O}^{\text {metal }}+\tilde{\mu}_{O}^{\text {metal }}\right)+K_{B} T \ln x_{O}^{\text {metal }}$

whilst $\tilde{\mu}_{\mathrm{SiO}_{2}}=\tilde{\mu}_{S i}+2 \tilde{\mu}_{O}$ in the liquid, which when rearranged (for a dissociation reaction) becomes equal the distribution coefficient

$$
K_{d}=\frac{x_{S i}^{m e t a l} x_{O}^{m e t a l}{ }^{2}}{x_{S i O_{2}}^{\text {silicate }}}=\exp \left(-\frac{\tilde{\mu}_{S i O_{2}}^{\text {metal }}-\tilde{\mu}_{S i O_{2}}^{\text {silicate }}}{k_{B} T}\right)
$$

allowing us to validate our thermodynamic model.

We focus on pressures and temperatures most relevant to the CMB (124 GPa and 4500-5500 K), as these are the most crucial for the evolution of the core, and also to avoid complications with changes in magnetic moment at shallower conditions. Simulations were run using the VASP code (Kresse and Furthmüller, 1996) in the canonical ensemble using a Nosé thermostat (Nosé, 1984) and with the Brillioun Zone sampled at the $\Gamma$ point. A timestep of 1 fs was used and runs lasted between 10 and 100 ps. The plane wave cutoff was set to $500 \mathrm{eV}$ and the projector augmented wave method (Kresse and Joubert, 1999) was used with the generalised gradient approximation functional PW91 (Perdew et al., 1992). The number of valence electrons and core radii for $\mathrm{Fe}, \mathrm{Si}$ and $\mathrm{O}$ were 14, 4 and 6, and 1.16, 0.7 and 0.08 Angstroms, respectively. Simulations contained between 148 and 160 atoms, depending on composition (reported in Table 1). 
187

188

\subsection{Thermodynamic Model}

The equilibrium constant $(K)$ describes a reaction at equilibrium

$$
K=\frac{\Pi_{i} a_{i}^{\alpha_{i}}}{\prod_{j} a_{j}^{\alpha_{j}}}=\frac{\Pi_{i} x_{i}^{\alpha_{i}}}{\Pi_{j} x_{j}^{\alpha_{j}}} \cdot \frac{\Pi_{i} \gamma_{i}^{\alpha_{i}}}{\Pi_{j} \gamma_{j}^{\alpha_{j}}}
$$

where $a_{i}$ are activities, $\alpha_{i}$ are reaction exponents, $x_{i}$ and $x_{j}$ are the molar concentration of reactants and $\gamma_{i}$ are activity coefficients. Experiments typically sample the abundance of reactants after the reaction has reached equilibrium, in this case $x_{S i}^{m e t a l}$ and $x_{S i}^{\text {silicate }}$. It is therefore convenient to use $K_{d}$ which describes the proportions of reactants at equilibrium, and is related to $K$ by

$$
K=K_{d} \cdot \frac{\Pi_{i} \gamma_{i}^{\alpha_{i}}}{\Pi_{j} \gamma_{j}^{\alpha_{j}}}
$$

$K_{d}$ takes different forms depending on the reaction pathway. Si might transfer between metal and silicate through dissociation, dissolution and exchange, which take the forms

$$
S i O_{2}^{\text {silicate }} \rightleftharpoons S i^{\text {metal }}+2 O^{\text {metal }}
$$

$$
\mathrm{SiO}_{2}^{\text {silicate }} \rightleftharpoons \mathrm{SiO}_{2}^{\text {metal }}
$$

and

$$
\mathrm{SiO}_{2}^{\text {silicate }}+2 \mathrm{Fe}^{\text {metal }} \rightleftharpoons 2 \mathrm{Fe} \mathrm{O}^{\text {silicate }}+\mathrm{Si}^{\text {metal }}
$$


189

for which $K_{d}$ is given respectively by

$$
K_{d}=\frac{x_{S i}^{m e t a l} x_{O}^{\text {metal }}{ }^{2}}{x_{S i O_{2}}^{\text {silicate }}}
$$

190

$$
K_{d}=\frac{x_{\mathrm{SiO}_{2}}^{\text {metal }}}{x_{\mathrm{SiO}_{2}}^{\text {silicate }}}
$$

191 or

$$
K_{d}=\frac{\left(x_{F e O}^{\text {silicate }}\right)^{2}}{\left(x_{F e}^{\text {metal }}\right)^{2}} \frac{x_{S i}^{\text {metal }}}{x_{S i O_{2}}^{\text {silicate }}} .
$$

${ }_{192}$ We discuss the choice of reaction for our model in our results (section 3). For a thermodynamic model, it is more useful to describe $K$ as the free energy 194 change of a reaction

$$
K=\exp \left(-\frac{\Delta F_{r}}{k_{B} T}\right)=\exp \left(-\frac{\Delta G_{r}}{k_{B} T}\right)=\exp \left(-\frac{\Delta H_{r}-T \Delta S_{r}+P \Delta V_{r}}{k_{B} T}\right)
$$

195

where $\Delta F_{r}$ is the Helmholtz free energy of reaction and $\Delta G_{r}$ is the equivalent Gibbs free energy change, representing conditions of constant volume $(V)$ and pressure $(P)$, respectively. $\Delta H_{r}, \Delta S_{r}$ and $\Delta V_{r}$ are the changes in enthalpy, entropy and volume with reaction, respectively, and $k_{B}$ is the Boltzmann constant. Eq. 14 is often written

$$
\log K=a+\frac{b}{T}+c \frac{P}{T}
$$




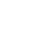

where $a, b$ and $c$ describe the entropy $(S)$, enthalpy $(H)$ and volume $(V)$ changes of reaction, respectively. This naming convention is adopted over traditional thermodynamic notation because these quantities are not exclusively represented by $a, b$ or $c$; entropy for example, will have a pressure and temperature dependence which is not captured by $a$ and so, in practice, these effects will be absorbed into $b$ and $c$. What cannot be absorbed into these parameters is the compositional dependence of the reaction.

Combining Eq. 7 and 15 gives the model

$$
\log K_{d}=a+\frac{b}{T}+c \frac{P}{T}-\sum_{i}\left(\log \gamma_{i}\right)+\sum_{j}\left(\log \gamma_{j}\right)
$$

which is fit to calculated $K_{d}$ from previous experimental partitioning results via a least squares approach. The best-fit parameters are used to calculate the equilibrium concentration of $\mathrm{Si}$ for a dissociation reaction (which we later show to be favourable) in the liquid metal using

$$
x_{S i}^{\text {metal }}=\exp \left(a+\frac{b}{T}+c \frac{P}{T}+\log x_{S i O_{2}}^{\text {silicate }}-2 \log x_{O}^{\text {metal }}-2 \ln \gamma_{O}-\ln \gamma_{S i}\right) .
$$

Our model describes Si partitioning between silicate liquid representing the molten mantle and an iron-rich liquid representing the liquid core. Following Badro et al. (2018), we use the interaction parameter model of Ma 
215

216

(2001) to define the activity coefficients of the solutes, $\gamma_{S i}$ and $\gamma_{O}$ by

$$
\begin{aligned}
\ln \gamma_{i} & =\ln \gamma_{F e}+\ln \gamma_{i}^{0}-\epsilon_{i}^{i} \ln \left(1-x_{i}\right) \\
& -\sum_{j=1(i \neq j)}^{N-1} \epsilon_{i}^{j} x_{j}\left(1+\frac{\ln \left(1-x_{j}\right)}{x_{j}}-\frac{1}{1-x_{i}}\right) \\
& +\sum_{j=1(i /=j)}^{N-1} \epsilon_{i}^{j} x_{j}^{2} x_{i}\left(\frac{1}{1-x_{i}}+\frac{1}{1-x_{j}}+\frac{x_{i}}{2\left(1-x_{i}\right)^{2}}-1\right),
\end{aligned}
$$

where

$$
\ln \gamma_{i}^{0}(T)=\left(\ln \gamma_{i \mathrm{a}}^{0}+\frac{\ln \gamma_{i \mathrm{~b}}^{0}}{T}\right) \frac{T_{0}}{T}
$$

and $T_{0}=1873 \mathrm{~K}$. The activity coefficient of the solvent $\left(\gamma_{\mathrm{Fe}}\right)$ is described in the supplementary information. This method does not include activities for the silicate liquid. For a partitioning model to be completely general, all elements in experiments and calculations must be represented in the $\epsilon$ and $\gamma$ parameter suite. This is not practical due to the inherent complexity and lack of sufficient experiments to fit for uniquely complex compositions. Du et al. (2017) make the assumption that only the relative proportions of each component in the metal influence partitioning with no accounting for interactions. This essentially assumes ideal mixing but requires few parameters. Badro et al. (2018) implement the interaction parameter model, focusing on high pressure and temperature data. The small number of studies at these conditions limits the ability to resolve all interaction parameters. We choose to include interactions for $\mathrm{C}, \mathrm{O}, \mathrm{Si}, \mathrm{S}$, and $\mathrm{Mg}$ (the same elements chosen by Badro et al. (2018)) as these are the commonly considered light el- 
ements in the outer core. Scatter in our distribution will always remain in the best-fitting model because not all elements are represented and experimental uncertainty is inevitable.

Our dataset includes 16 studies spanning conditions from 0 to $100 \mathrm{GPa}$ and 1754 to $5700 \mathrm{~K}$, the complete details of which or provided in the supplementary information. This wide range of conditions gives a sufficient number of data points to resolve all interaction parameters in our model. Experimental apparatus include piston cylinder presses, multi anvil presses and diamond anvil cells and values of $\log K$ range from -13 to -3 . Because on the wide range of setups, conditions and chemistry our model must be flexible in order to capture all of them. This flexibility makes our model suitable for a wide range of core chemistries as well as core formation processes at lower pressures.

\section{Results}

In Table 1 we report our ab inito results of the calculated excess chemical potential of $\mathrm{SiO}_{2}$ at $5500 \mathrm{~K}$ and $4500 \mathrm{~K}$, both at $124 \mathrm{GPa}$. We show consistency with experimental $K_{d}$ at comparable $T$ and $P$ in Fig. 1. Differences in compositions make direct comparison of $K_{d}$ difficult but indicate an overall agreement in trends. The highest PT experiments (Badro et al., 2016, 2018; Suer et al., 2017) compare well to our results especially at $4500 \mathrm{~K}$ which lies within the scatter of experiments at similar temperatures. Strong temperature dependence and a weaker pressure effect, especially above $50 \mathrm{GPa}$, 
is commensurate with an entropy dominated, configurational change in the iron-rich liquid.

\begin{tabular}{ccc}
\hline Metal composition & $\mathrm{Fe}_{149} \mathrm{O}_{8}$ & $\mathrm{Fe}_{149} \mathrm{O}_{8}$ \\
Silicate composition & $\mathrm{Mg}_{28} \mathrm{Fe}_{4} \mathrm{Si}_{32} \mathrm{O}_{96}$ & $\mathrm{Mg}_{28} \mathrm{Fe}_{4} \mathrm{Si}_{32} \mathrm{O}_{96}$ \\
$\mathrm{P}$ & $124 \mathrm{GPa}$ & $124 \mathrm{GPa}$ \\
$\mathrm{T}$ & $5500 \mathrm{~K}$ & $4500 \mathrm{~K}$ \\
$\delta \tilde{\mu}_{\text {SiO }}$ & $0.33(9) \mathrm{eV}$ & $1.37(26) \mathrm{eV}$ \\
$K_{d}^{\text {dissociation }}$ & $0.50_{+0.09}^{-0.09}$ & $0.029_{+0.014}^{-0.028}$ \\
$K_{d}^{\text {dissolution }}$ & $0.18_{+0.03}^{-0.03}$ & $0.011_{+0.005}^{-0.010}$ \\
$K_{d}^{\text {exchange }}$ & $0.19_{+0.04}^{-0.03}$ & $0.011_{+0.011}^{-0.005}$ \\
\hline
\end{tabular}

Table 1: Details of ab initio simulations and calculated results of excess chemical potential and distribution coefficient. Compositions are shown as the number of atoms for each species present in the calculations.
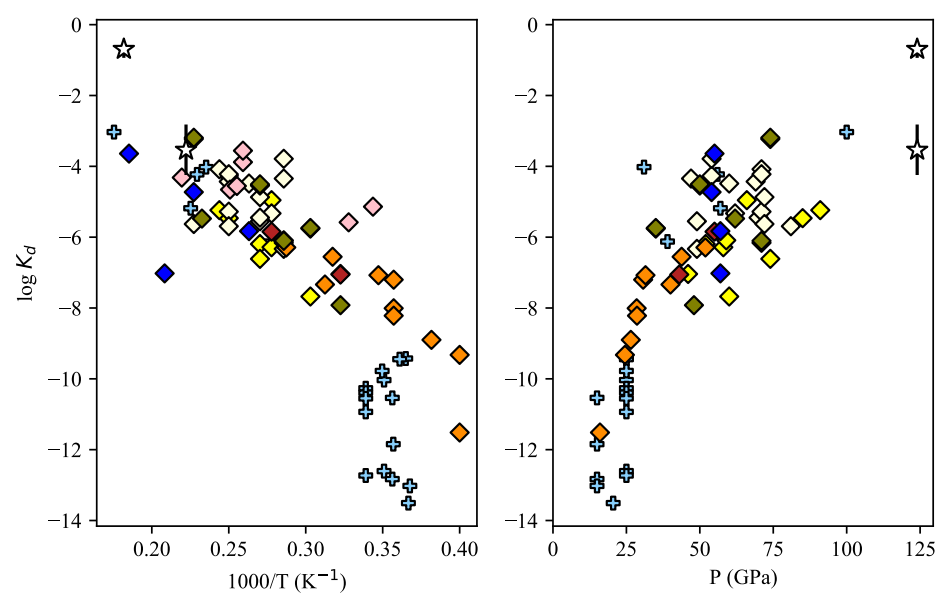

Figure 1: Comparison of distribution coefficients $\left(K_{d}\right.$, Eq.11) calculated from a high pressure and temperature subset of our chosen experimental partitioning dataset (coloured symbols: diamonds are diamond anvil cell (DAC) studies and pluses are studies with both DAC and multi-anvil press (MAP) experiments) with ab initio results of this study (white stars). Low PT studies are omitted here to focus on conditions similar to our calculations. Studies included are: Fischer et al. (2015) (light blue), Suer et al. (2017) (yellow), Badro et al. (2016) (brown), Badro et al. (2018) (cream), Chidester et al. (2017) (blue), Hirose et al. (2017) (pink) and Bouhifd and Jephcoat (2003) (orange). 
Having established the validity of our calculations, we now construct a thermodynamic model to describe partitioning behaviour but must first choose a reaction which governs the transfer of Si (Eq.s 8, 9 and 10). We do this by evaluating the quality of fit for our model to experimental partitioning for each reaction. The dissociation reaction proves to be the superior fit $\left(\chi^{2}=3.43 \times 10^{-5}\right)$ when compared to dissolution $\left(\chi^{2}=2.60\right)$ and exchange $\left(\chi^{2}=2.22 \times 10^{-3}\right)$. Badro et al. (2018) found dissociation and dissolution to be similar in quality for $\mathrm{Mg}$ transfer (outperforming exchange) but opted for dissociation as oxides are not expected in the metallic liquid. Helffrich et al. (2020) propose an alternate exchange reaction $\left(\right.$ SiO $\left.\mathrm{O}_{2}^{\text {silicate }}+2 M g^{\text {metal }} \rightleftharpoons 2 M g O^{\text {silicate }}+S i^{\text {metal }}\right)$ which we do not consider, however, our inclusion of $\mathrm{Mg}$ in the interaction parameters accommodates any importance it may have. For consistency with metalic liquid behaviour and superior quality of fit, our model is based on the dissociation reaction (Eq. 11).

Our model includes interaction parameters for $\mathrm{C}, \mathrm{O}, \mathrm{Si}, \mathrm{S}$ and $\mathrm{Mg}$, and has the possibility to include all interactions between them, something not resolvable in previous studies. In Fig. 2 we show that all interactions have an influence on the quality of the thermodynamic model. By far the most influential are those between $\mathrm{O}-\mathrm{O}, \mathrm{Si}-\mathrm{Si}$ and $\mathrm{Si}-\mathrm{O}$ followed by the remaining oxide interactions. Whilst unsurprising, this highlights the importance of oxygen content in the core and the predominant configurations of the liquid. We examine the relative quality of fit in our model for different numbers of 
interaction parameters included. The optimal combination for each number included is the combination of interaction parameters which produces the model with the lowest $\chi^{2}$ when fit to our dataset (when compared to all other permutations). Du et al. (2017) use a model which only considers self-interaction of chemical species, Fig. 2 demonstrates that far fewer parameters can be used to achieve a similar quality of fit when the most influential interactions are considered. Similarly, we find that if parameters are assumed to be important without prior knowledge (e.g. $\epsilon_{O}^{S i}$ and $\epsilon_{O}^{M g}$ ) the quality of the model fit can be dramatically worse $(\sim 10 \times)$ than the optimal combination. For a simple implementation we recommend a minimum model containing 9 of the most crucial interactions (detailed in the supplementary information) as this achieves a near identical quality to our complete model. For completeness, we include all 15 parameters in our model throughout this study. 


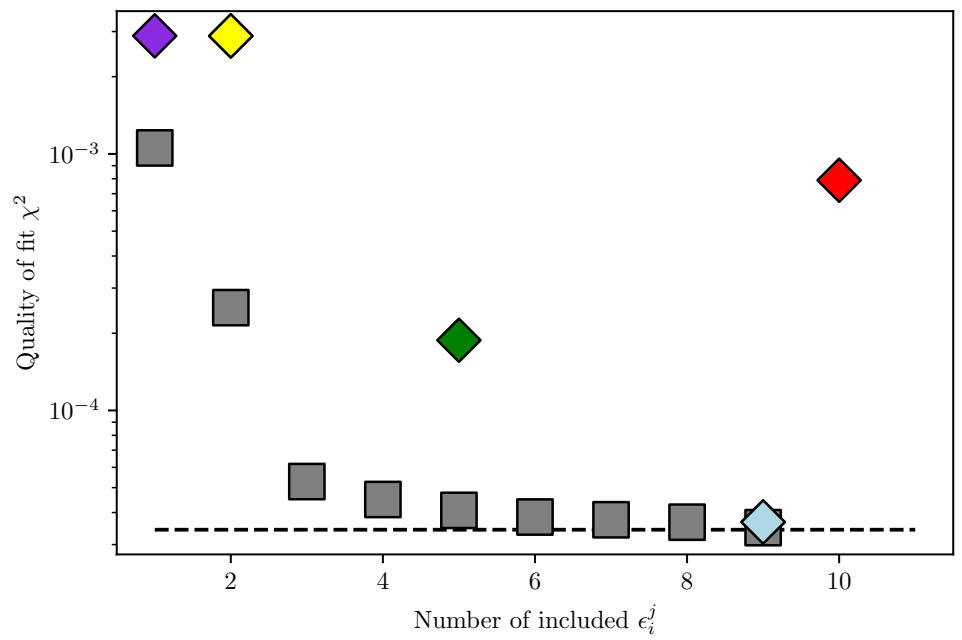

Figure 2: Quality of fit $\left(\chi^{2}\right)$ to experimental partitioning for the number of interaction parameters included in our model out of the possible pair permutations of $\mathrm{C}, \mathrm{O}, \mathrm{Si}, \mathrm{S}$ and $\mathrm{Mg}$. Grey squares represent the best fit of the optimal combination of $\epsilon_{i}^{j}$ parameters, coloured diamonds show the quality of fit for some conventionally adopted interactions. Purple is $\epsilon_{O}^{S i}$ only, yellow is $\epsilon_{O}^{S i}$ and $\epsilon_{O}^{M g}$, green is all self interactions $\left(\epsilon_{i}^{j=i}\right)$, light blue is all interactions involving oxygen and silicon $\left(\epsilon_{O}^{j}, \epsilon_{S i}^{j}\right)$ and red is all unequal interactions $\left(\epsilon_{i}^{j \neq i}\right)$. The black dashed line shows the quality achieved through inclusion of all $15 \epsilon_{i}^{j}$ parameters.

In Fig. 3 compare the variation of $K$ from the compositions of our dataset with and without the inclusion of $\gamma_{i}$. The inclusion of $\gamma_{i}$ gives an improvement to the quality of our model over assumed ideal behaviour $\left(\gamma_{i}=0\right)$. Indeed, neglecting these terms forces entropic effects associated with composition into temperature dependence ( $b$ in Eq. 15), giving a falsely large gradient $\left(-44 \frac{\log K}{1000 / T}\right.$ vs $\left.-26 \frac{\log K}{1000 / T}\right)$. Hirose et al. (2017) find a gradient of $-35 \frac{\log K}{1000 / T}$, intermediate to our findings with and without interaction parameters, inline with their use of only Si and O interaction parameters. Helffrich et al. (2020) use the interaction parameters of Fischer et al. (2015) for Si, who find -31 
301

$\frac{\log K}{1000 / T}$

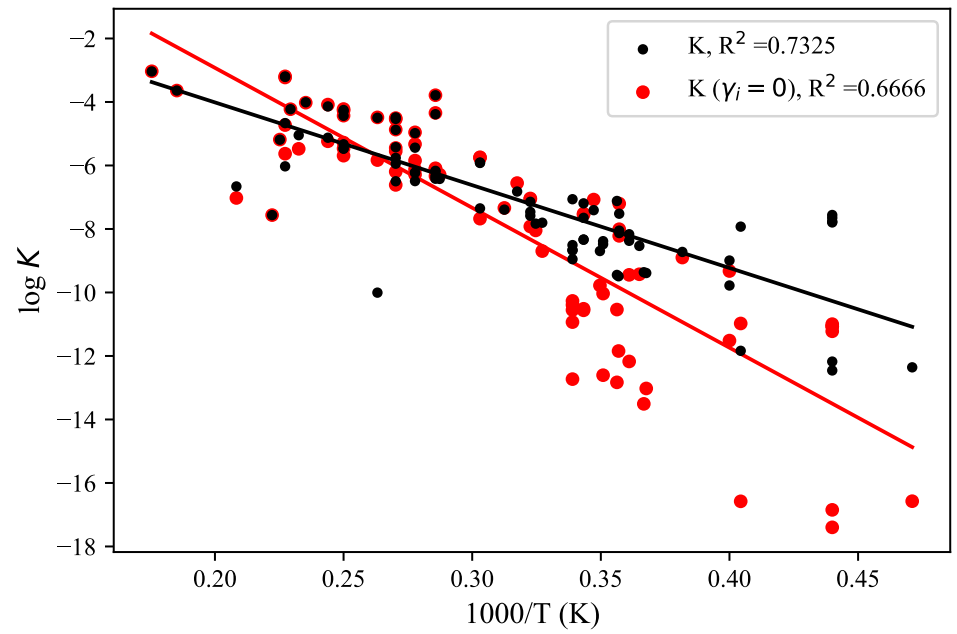

Figure 3: Equilibrium constants for our dataset with experiments where Si is not detected $\left(x_{S i}^{\text {metal }}=0\right)$ removed. Red points show activities set to zero (equivalent to ideal mixing) and black show our complete model. The improved fit achieved by including interaction parameters is shown by the $R^{2}$ value of the fitted $\operatorname{lines}\left(\log K=m \frac{1000}{T}+d\right)$. Due to the diversity of our dataset, small number of outlying points adversely effect our fit quality.

Despite the wide range of conditions spanned by experiments in our dataset, we find that temperature dependence of partitioning is well approximated by $\log K=m \frac{1000}{T}+d$, particularly for simpler compositions (few elements not included in our parameter set). This gives us confidence that our model sufficiently captures the complexity of Si transfer for core formation and evolution conditions.

Fig. 4 shows how Si solubility depends on the concentration different light elements in the liquid metal at a single TP point. We find that the equilibrium fraction of $\mathrm{Si}$ is strongly limited by the $\mathrm{O}$ content of the liquid 
311

312

313

314

315

316

317

318

319

320

321

322

creating an important excluding effect on composition in agreement with Hirose et al. (2017) and Helffrich et al. (2020). Because the concentration of $\mathrm{O}$ in the metal adversely effects the solubility of $\mathrm{Si}$ in the metal, there is little compositional space for them to coexist in significant amounts, especially in high concentrations. We find that for equal proportions of $\mathrm{Si}$ and $\mathrm{O}$ to be present in the metal, only $\sim 1 \mathrm{wt} \%$ of each can be hosted in an iron-rich liquid at core conditions. $\mathrm{C}$ also limits the solubility of $\mathrm{Si}$, but to a far lesser degree, whilst S has little appreciable effect. Mg has a weak but opposite effect on Si solubility compared to $\mathrm{O}$, where increasing the concentration of $\mathrm{Mg}$ (for moderate concentrations) in the liquid metal allows higher Si concentration.

We note that these influences are complex and depend themselves on the liquid composition. 


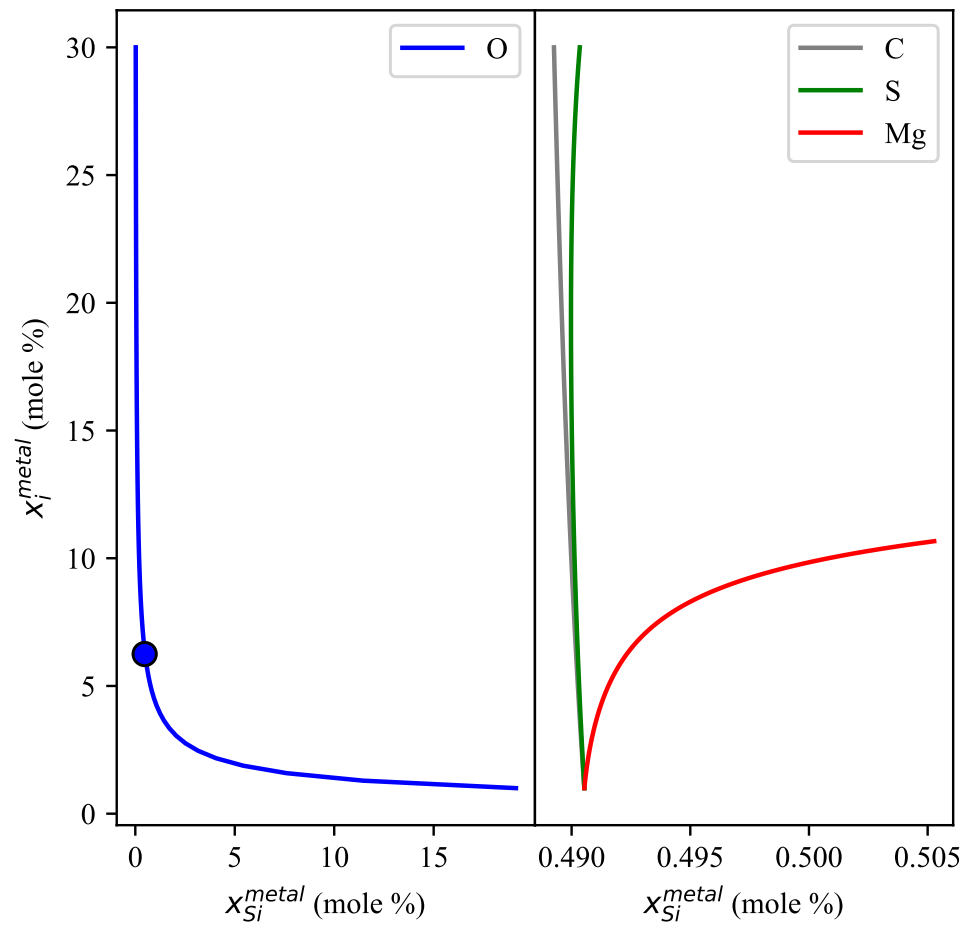

Figure 4: Stable mole fraction of $\mathrm{Si}$ in the liquid metal at $4500 \mathrm{~K}$ and $124 \mathrm{GPa}$ in equilibrium with pyrolite liquid for different concentrations of $\mathrm{O}$ (blue, left), $\mathrm{C}$ (grey, right), $\mathrm{S}$ (green, right) and $\mathrm{Mg}$ (red, right). A standard solvent composition of $\mathrm{Fe}_{0.9375-\mathrm{x}_{S i}} \mathrm{Si}_{\mathrm{x}_{S i}} \mathrm{O}_{0.0625} \mathrm{~S}_{0} \mathrm{C}_{0} \mathrm{Mg}_{0}$ is applied for each element when not being varied. Prediction of $x_{S i}$ from ab initio $K_{d}$ is shown as a blue circle.

\section{Discussion}

Our model robustly allows us to calculate the maximum stable fraction of Si in iron-rich liquids at conditions from the upper mantle to the CMB. By including the interactions of $\mathrm{C}, \mathrm{O}, \mathrm{S}$ and $\mathrm{Mg}$ we are able to describe a wide range of liquid compositions which is necessary as the initial composition of 
the core is poorly constrained. We now use our model to explore the chemical evolution of the core.

\subsection{Partitioning of Silicon}

We apply our model to the core using Eq. 17 at fixed $P$ as a function of $T$ to estimate Si concentration of the core given an initial core composition and a mantle $\mathrm{SiO}_{2}$ concentration. The experiments to which our model is fit all examine a liquid silicate reacting with a liquid metal. For the Earth, this would have been the magma ocean interacting with the liquid core, when the mantle was subject to super solidus CMB temperatures. We apply our model, despite it's assumption of liquid-liquid interaction at all temperatures including those below the solidus of $\mathrm{SiO}_{2}$ (e.g. Usui and Tsuchiya (2010)). We assume the core to be well mixed throughout for convenience, although this is not a certainty. The core may have initially hosted a chemically stratified layer in contact with the CMB (Landeau et al., 2016; Jacobson et al., 2017; Davies et al., 2020), which would likely have delayed the onset of precipitation. For simplicity we neglect any stratified layers and instead treat the core as compositionally homogeneous, and changes with radius to be purely adiabatic (Davies, 2015; Nimmo, 2015b; Labrosse, 2015). Stratified layers will inhibit precipitation as the bulk mantle and core are separated and instead must interact via diffusion through the later. As a proxy for a chemically stratified layer or an initially undersaturated core, we consider cases of delayed onset precipitation, where the initial concentration of $\mathrm{Si}$ in 
the metal is undersaturated.

When considering the interface between the bulk core and bulk mantle, if the core is considered to be in equilibrium with the magma ocean initially (this is a simple case, Davies et al. (2020) consider a more complex scenario), all dissolved light elements must be thermodynamically stable. Upon cooling, the equilibrium concentration of Si will decrease and the liquid becomes thermodynamically unstable, requiring some precipitation to re-establish equilibrium. The $\mathrm{CMB}$ is the coolest region of the core and so becomes depleted with respect to the rest of the core. Residual liquids in the outermost core are dense and will sink, becoming mixed with the rest of the liquid core. The newly well-mixed core will remain thermodynamically unstable, forcing the process to continue until the entire core is at the CMB equilibrium concentration (Badro et al., 2016), therefore this concentration controls the composition of the entire core. This assumes that the mixing rate of the core is many times faster than the cooling rate, where the cooling rate is $\sim 10^{2} \mathrm{~K} \mathrm{Gyr}^{-1}$ (Gubbins et al., 2015) and the advection time (which defines the rate of mixing) of the core is $\sim 10^{2} \mathrm{yr}$ (advection time $=\frac{L}{U}$ where $L$ is the length scale of the core, $3486 \mathrm{~km}$, and $U$ is the fluid velocity of the

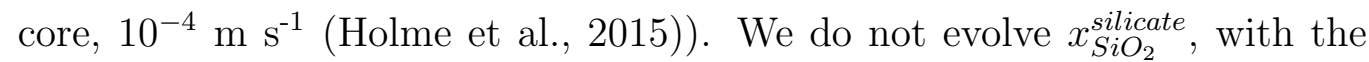
assumption that precipitation does not alter the composition of the mantle significantly near the CMB because the mantle is large and entrains precipitates perfectly (Buffett and Seagle, 2010) and the total Si content of the core is small by comparison. 
Under these assumptions we evaluate Si solubility in the core based on the CMB pressure and evolving temperature and core composition. Due to the strong $\mathrm{O}$ dependence of Si solubility in our model, we first examine a fixed oxygen concentration of $2 \mathrm{~mol} \%$ such that the initial Si concentration can stably be within the range of compositions predicted from core formation models (Badro et al. (2015); Rubie et al. (2015); Fischer et al. (2017), see Fig. 5). We represent the possible silicate melt compositions $\left(x_{\mathrm{SiO}_{2}}^{\text {silicate }}\right)$ simply with two compositional scenarios: scenario $1 ; \mathrm{SiO}_{2}$ and scenario 2; pyrolite. Scenario 1 has a growing, stable layer of pure precipitate $\left(x_{\mathrm{SiO}_{2}}^{\text {silicate }}=1.0\right.$, dashed line, Fig. 5) which the overlying mantle does not alter the composition of (diffusion into the layer is ignored). Scenario 2 represents a mantle which quickly entrains any precipitate from the core and is large enough not to be diluted so the core always interacts with a pyrolite composition $\left(x_{\mathrm{SiO}_{2}}^{\text {silicate }}=0.387\right.$, Fig. 5 solid line $)$. Fig. 5 compares the temperature dependent Si solubility for both scenarios and includes the uncertainty from the fitting parameters in our model. In scenario 1 a more Si enriched core with long lived precipitation is possible and final concentrations are approximately compatible with the compositions needed to produce the inner core density jump in the range $0.6-0.8 \mathrm{~g} \mathrm{~cm}^{-3}$ (Davies et al., 2015). Overall high Si solubility in the core means that precipitation rates are low compared to more moderate silicate compositions used by Hirose et al. (2017) and Mittal et al. (2020). The initial Si concentration for constant precipitation in scenario 1 far exceeds what is suggested to be available from accretionary models (Badro 
et al., 2015; Rubie et al., 2015; Fischer et al., 2017), although this would be lower for a higher core $\mathrm{O}$ concentration. In scenario 2 solubility is reduced at all temperatures and the available precipitation rates are halved compared to scanario 1, falling further from those found by previous studies, however, low temperature concentrations prove to be similar. Our model shows that ancient core compositions may have been less Si enriched than required by some experimental studies. In these simple cases, stable Si concentration fails to match compositions compatible with the inner core density jump, however the budget for this regime is within the estimates of post-accretion core $\mathrm{Si}$ content. In reality we expect scenario 1 to be unlikely because a precipitate layer should not survive mantle entrainment (Buffett and Seagle, 2010) and scenario 2 to be more likely because with a more complex composition, additional light elements in low concentration, present day seismic constraints could be satisfied whilst still having consistent precipitation to power the geodynamo.

Within the uncertainties on the initial compositions estimated from core formation models (Badro et al., 2015; Rubie et al., 2015; Fischer et al., 2017) our model shows that precipitation may have occurred for the duration of Earth history or not at all. This uncertainty also suggests that a large fraction of Si could be precipitated early in Earth history, implying that the core can be in disequilibrium with the bulk mantle during formation (Rubie et al., 2015), or that the core would not have been able to reach these compositions. Equilibrium of bulk core and mantle compositions should therefore not be a 
419

420

421

422

423

424

425

426

427

constraint on the initial condition of the core.

Fig. 5 also presents rates of precipitation (right) ranging from $10^{-7} \mathrm{wt} \%$ $\mathrm{K}^{-1}$ to $10^{-4} \mathrm{wt} \% \mathrm{~K}^{-1}$ for a set of compositions; 2,4 and $10 \mathrm{~mol} \% \mathrm{O}$, where the initial $\mathrm{Si}$ concentration is defined by the maximum stable solution. These compositions are chosen to capture previously considered values, although the unconstrained nature of core composition makes choosing a preferred option difficult. We observe weak temperature dependence and strong $x_{O}^{\text {metal }}$ and $x_{\mathrm{SiO}_{2}}^{\text {silicate }}$ dependence of precipitation rate, with negative and positive influence respectively. 

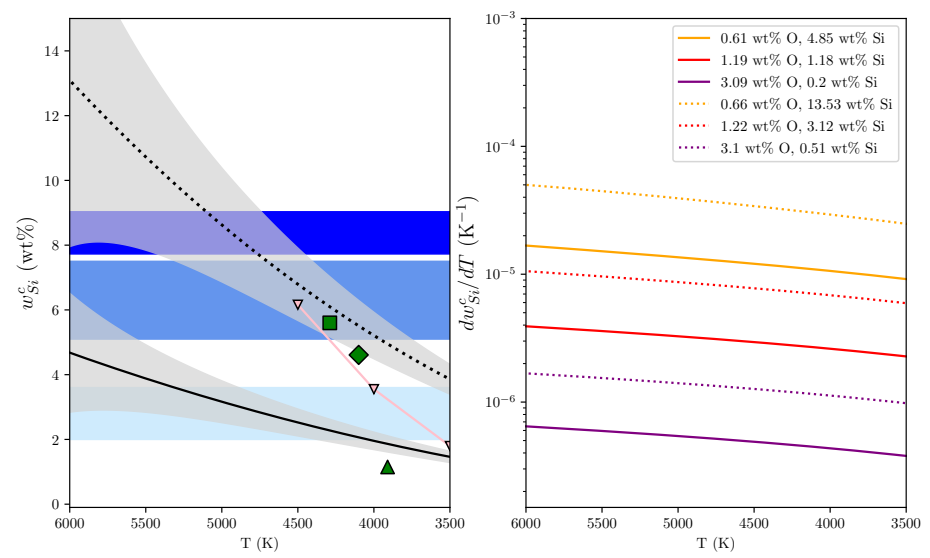

Figure 5: Left: Stable concentration of $\mathrm{Si}$ in the liquid metal. Our model is evaluated for partitioning with a pyrolite mantle (black solid line) and with a pure $\mathrm{SiO}_{2}$ layer at the CMB (black dashed line), both at $124 \mathrm{GPa}$. Grey shaded envelopes are uncertainties from the fitting parameters of our model. Horizontal shaded regions are estimated initial core compositions of Rubie et al. (2015) (dark blue), Fischer et al. (2017) (mid blue) and Badro et al. (2015) (light blue). Davies et al. (2015) estimate present-day core composition (within an Fe-Si/S-O core) based on the inner core density jump (green square, diamond and triangle for density jumps of $0.6,0.8$ and $1.0 \mathrm{~g} \mathrm{~cm}^{-3}$, respectively). LH-DAC experiments of Hirose et al. (2017) are shown as red symbols. Right: Precipitation rates from our model for a range of initial $\mathrm{Si}$ and $\mathrm{O}$ concentrations in the core, partitioning with pyrolite (solid lines) and $\mathrm{SiO}_{2}$ (dotted lines).

\subsection{Thermal evolution of the core}

To investigate the effect of precipitation on the thermal evolution of the core we combine two classical models; the Greenwood et al. (2021); Davies and Greenwood (2022) model of the core and the Driscoll and Bercovici (2014) model of the solid mantle. These parameterisations of the deep Earth are coupled at the $\mathrm{CMB}$ where the mantle defines the heat transport across the $\mathrm{CMB}$ and the core defines the temperature of the CMB. For the core, energy balance is used to evolve the core temperature and composition along- 
side entropy balance to evaluate the entropy production due to the magnetic field. A moderately high conductivity scenario is considered, applying a constant $70 \mathrm{~W} \mathrm{~m}^{-1} \mathrm{~K}^{-1}$ thermal conductivity in the core. A solid mantle is included, however, we do not include a separate magma ocean to minimise the contributions to geodynamo power such that precipitation effects are clearly distinguishable and to minimise the number of uncertain parameters. This setup is not fully consistent with our thermodynamic model which is based on liquid-liquid reactions in both experiments and calculations. Due to the high CMB temperatures present in many of our models, we envisage (purely for convenience) a simplistic, persistent thin melt layer at the base of the mantle, with negligible latent heat release and an equal partitioning of radiogenic elements (which we do not expect to be the case in reality) with the overlying mantle. We assume that the core is mixed thoroughly on timescales far shorter than the timestep of our simulation such that the liquid core has no compositional variation nor stable layers.

To balance energies in the core, we follow Davies (2015) where, if small terms are ignored, the heat flow across the $\mathrm{CMB}\left(Q^{c m b}\right)$ is

$$
Q^{c m b}=Q_{s}+Q_{L}+Q_{p}+Q_{g}
$$

where $Q_{s}$ is the secular heat stored in the core and $Q_{L}$ is the latent heat release due to inner core growth. $Q_{p}$ is the gravitational energy from mixing 
the dense, iron-rich residual liquids post precipitation across the outer core

$$
Q_{p}=\int_{\infty} \psi \rho \alpha_{p p t}^{i}\left[C_{p p t}\left(\frac{d T_{c m b}}{d t}\right)\right] d V_{c}
$$

where $\rho$ is density, $\alpha_{p p t}^{i}$ is expansivity, $\psi$ is gravitational potential, $C_{p p t}$ is the precipitation rate (see Fig. 5), $t$ is time and $V_{c}$ is volume of the liquid core. $Q_{g}$ is the gravitational power generated from the preferential partitioning of O into the liquid upon freezing. We assume Si to partition evenly between the solid and liquid core (Alfè et al., 2002) such that the growth of the inner core has no effect on the Si concentration of the liquid core. Gubbins et al. (2004) show that the entropy budget of the core can be balanced by

$$
E_{j}+E_{\alpha}+E_{k}=E_{s}+E_{L}+E_{p p t}+E_{g}
$$

where $E_{\alpha}$ is the entropy due to barodiffusion throughout the core which is negligible (Gubbins et al., 2004; Davies, 2015) and so is ignored. $E_{k}$ is the entropy from thermal conduction and the other terms follow the same notation as their energy counterparts.

If when evaluated, these entropy sources produce a positive $E_{j}$, the geodynamo can be sustained. This presents the difficulty in a high thermal conductivity core, the entropy balance now has a far larger $E_{k}$, taking power from $E_{j}$, and so to sustain a magnetic field before inner core growth, one or more of the r.h.s terms must be increased. Because time before inner core nucleation excludes the influence of $E_{L}$ and $E_{g}$, a more rapidly cooling core 
473

474

475

$\left(E_{s}\right)$ or precipitation $\left(E_{p p t}\right)$ are needed.

We vary the upper to lower mantle viscosity ratio $\left(f_{\text {visco }}\right)$ and initial CMB temperature $\left(T_{c m b}^{t=0}\right)$ to regulate the core temperature such that the final state of our models matches constraints of the present-day core. These constraints are: the inner core radius agreeing with present-day value of $1221 \mathrm{~km}$, a present-day mantle convective heat flow of 39 TW (Jaupart et al., 2007), a mid-mantle temperature of $2320 \mathrm{~K}$ and a positive entropy from ohmic dissipation $\left(E_{j}\right)$ for all time preceding inner core nucleation.

Table 2 provides the setup for our evolution cases. We investigate high (20 mol\%) and low (2 mol\%) initial oxygen compositions of the liquid core and a pyrolite mantle composition. Each of these initial compositions is evolved under conditions of initially over and undersaturated Si content and with $\left(\alpha_{p p t}^{i} \neq 0\right)$ and without $\left(\alpha_{p p t}^{i}=0\right)$ the convective power of precipitation included. 


\begin{tabular}{ccccccc}
\hline $\begin{array}{c}\text { Symbol } \\
\text { Units }\end{array}$ & $\begin{array}{c}\mathrm{O} \\
\mathrm{mol} \%\end{array}$ & $\begin{array}{c}\mathrm{Si} \\
\mathrm{mol} \%\end{array}$ & $\alpha_{p p t}^{O}$ & $\alpha_{p p t}^{S i}$ & $\begin{array}{c}T_{c m b}^{t=0} \\
\mathrm{~K}\end{array}$ & $f_{\text {visco }}$ \\
\hline $\mathrm{A}$ & 20 & 0.123 & 0.0 & 0.0 & 5500 & 0.23 \\
$\mathrm{~B}$ & 20 & 0.061 & 0.0 & 0.0 & 5500 & 0.23 \\
$\mathrm{C}$ & 2 & 11.9 & 0.0 & 0.0 & 6600 & 20 \\
$\mathrm{D}$ & 2 & 6.0 & 0.0 & 0.0 & 5900 & 14 \\
$\mathrm{~A}^{\mathrm{P}}$ & 20 & 0.123 & 1.1 & 0.87 & 5000 & 0.25 \\
$\mathrm{~B}^{\mathrm{P}}$ & 20 & 0.061 & 1.1 & 0.87 & 5700 & 0.19 \\
$\mathrm{C}^{\mathrm{P}}$ & 2 & 11.9 & 1.1 & 0.87 & 6000 & 8 \\
$\mathrm{D}^{\mathrm{P}}$ & 2 & 6.0 & 1.1 & 0.87 & 5800 & 9 \\
\hline
\end{tabular}

Table 2: Initial values for thermal evolution model runs $(\mathrm{A}-\mathrm{H})$ where all other quantities remain unchanged from Davies (2015) and Driscoll and Bercovici (2014) (for the core and mantle respectively) unless otherwise stated. Expansivity $\left(\alpha_{p p t}^{i}\right)$ is taken from Davies et al. (2015) and applies only to precipitation from the liquid core, non-zero values are applied to inner core growth for all cases. High $\left(\mathrm{A}, \mathrm{A}^{\mathrm{P}}, \mathrm{B}, \mathrm{B}^{\mathrm{P}}\right)$ and low $\left(\mathrm{C}, \mathrm{C}^{\mathrm{P}}, \mathrm{D}, \mathrm{D}^{\mathrm{P}}\right)$ oxygen cases are taken for $\mathrm{Si}$ over $\left(\mathrm{A}, \mathrm{A}^{\mathrm{P}}, \mathrm{C}, \mathrm{C}^{\mathrm{P}}\right)$ and under $\left(\mathrm{B}, \mathrm{B}^{\mathrm{P}}, \mathrm{D}, \mathrm{D}^{\mathrm{P}}\right)$ saturation with power from precipitation turned on $\left(\mathrm{A}^{\mathrm{P}}-\mathrm{D}^{\mathrm{P}}\right)$ and off $(\mathrm{A}-\mathrm{D})$.

Fig. 6 shows examples of the time evolution of inner core radius, midmantle potential temperature, CMB temperature, mantle convective heat flow, CMB heat flow and entropy production from ohmic dissipation. All cases where precipitation is not included fail to satisfy our constraints. This is because for the core to cool sufficiently to produce the inner core of present radius, they fail to consistently sustain a positive $E_{j}$. We find that including the energy and entropy effects of precipitation can maintain a positive $E_{j}$ for the majority of Earth history preceding inner core formation, also found to be the case by Hirose et al. (2017), producing an older inner core. We find that high initial oxygen concentration cases require $f_{\text {visco }}<1$ in order to grow the core to present-day size meaning the upper mantle is more viscous than the lower mantle. We do not expect this to be the case in reality (Rudolph et al., 


\section{5), highlighting the requirement for modest O content for Si precipitation}

to sustain a geodynamo whilst satisfying present-day constraints.
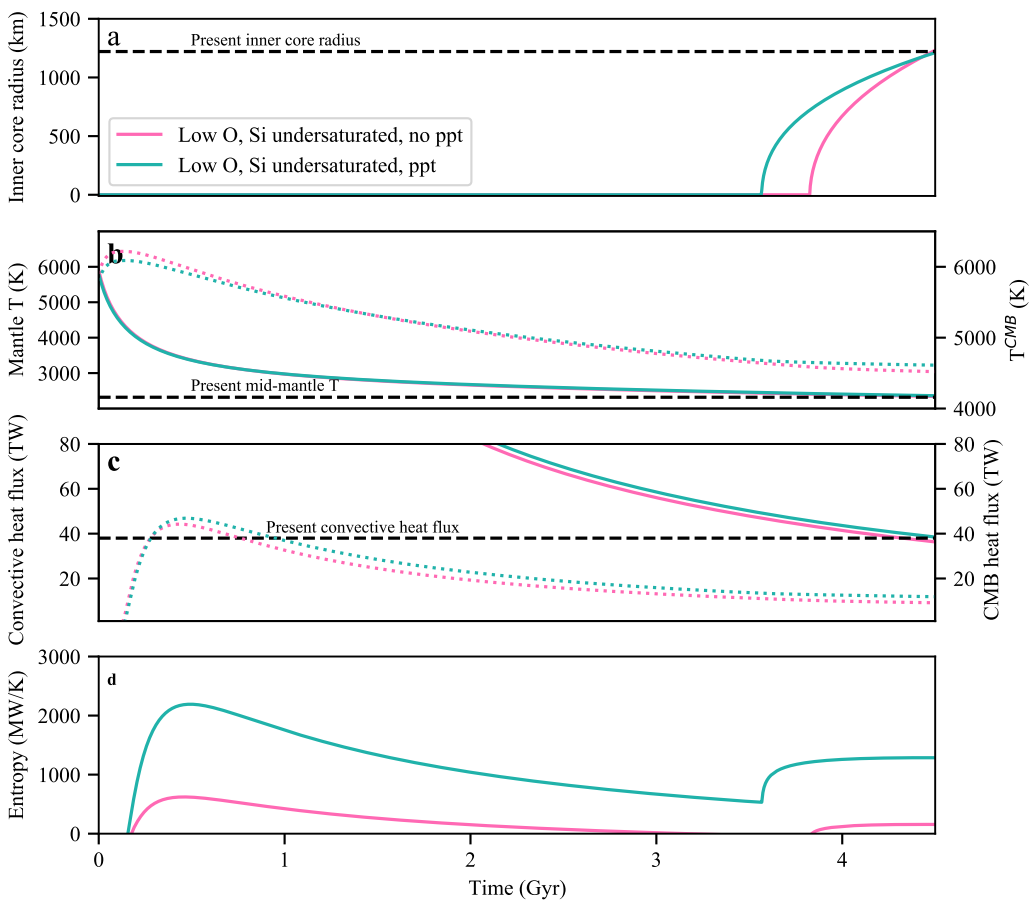

Figure 6: Thermal evolution of the Earth's core with an initial condition of low O concentration (2 mol\%) and Si saturation with inclusion (teal) and exclusion (pink) of power and entropy of precipitation (cases $\mathrm{D}$ and $\mathrm{D}^{\mathrm{P}}$ from Table 2, respectively). Inner core radius (a), mid-mantle potential temperature (b, left, solid lines) and CMB temperature (b, right, dotted lines), convective mantle heat flux (c, left, solid lines) and CMB heat flow (c, right, dotted), and core entropy from ohmic dissipation (d). Black dashed lines show present-day target values.

We show the outcomes of thermal history cases from Table 2 in Fig. 7. When $\mathrm{Si}$ is initially saturated and $\alpha_{p p t}^{S i}=0$ (cases A and C) a hotter core is required for the low $\mathrm{O}$ case and $Q_{C M B}$ is lower due to a larger $f_{\text {visco. }}$. The 
higher values of $f_{\text {visco }}$ are needed in order for the inner core to not grow too large by $4.5 \mathrm{Ga}$ whilst in the high $\mathrm{O}$ case low temperatures are needed to freeze the inner core due to further melting point depression. When $Q_{p} \neq 0$ (again for Si saturated initial conditions, cases $\mathrm{A}^{\mathrm{P}}$ and $\mathrm{C}^{\mathrm{P}}$ ) cooling rates are lower and the inner core is $\sim 300$ Myrs older for O poor conditions (more $\mathrm{Si}$ is available to precipitate). For cases of initial Si undersaturation (B, $\mathrm{B}^{\mathrm{P}}, \mathrm{D}, \mathrm{D}^{\mathrm{P}}$; meaning precipitation is delayed), a similar core temperature is needed both with and without precipitation, however, in all compositional configurations the inner core is older with the precipitation power (Hirose et al., 2017). When $\mathrm{Si}$ is initially saturated, including $Q_{p}$ allows lower cooling rates and an older inner core $\left(\mathrm{c}^{\mathrm{P}}=879 \mathrm{Myrs}\right)$, however the cooling rate is slightly lower in $Q_{p} \neq 0$ cases where $\mathrm{Si}$ is initially undersaturated (and precipitation is therefore delayed). Whilst all cases we considered are able to produce a geodynamo within the first 2 Gyrs, cases where $E_{p p t}=0$ are unable to produce a magnetic field from $\sim 800$ Myrs prior to inner core formation (crossed symbols, Fig. 7). 


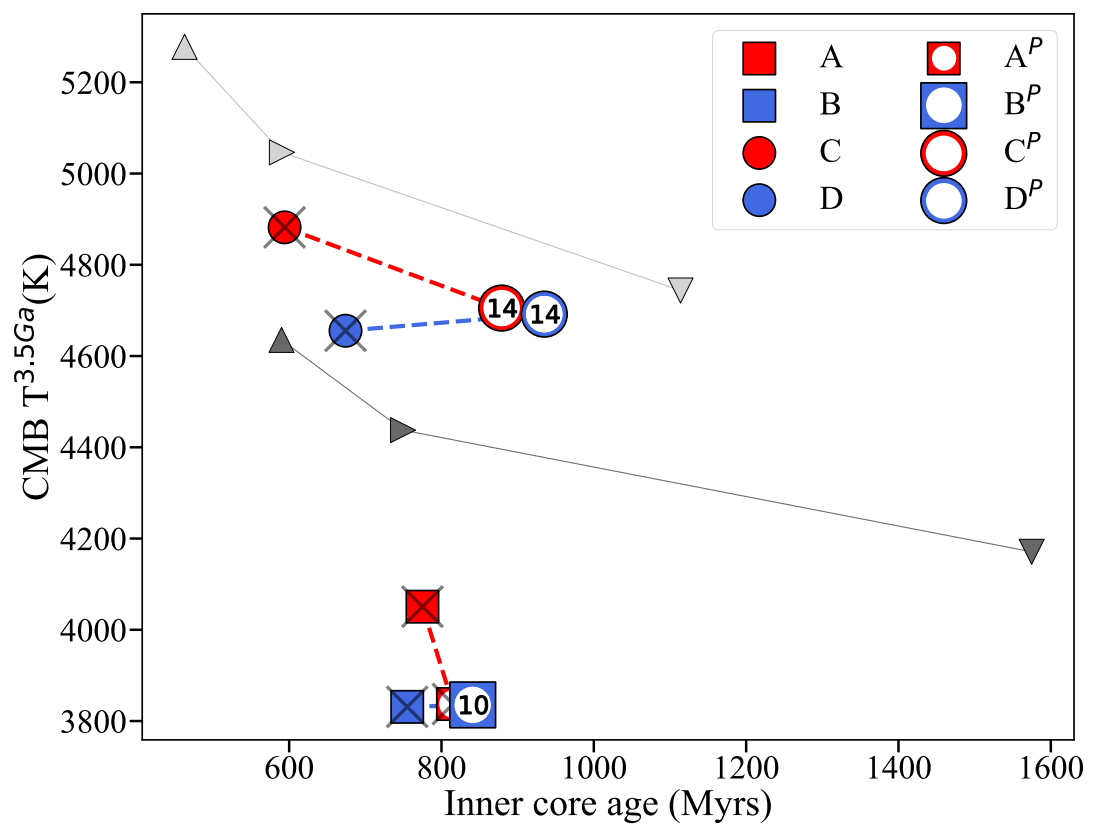

Figure 7: Inner core age and core temperature at 3.5 Ga for our model (coloured symbols) with and without convective power from precipitation (connected by dashed lines). Initial $\mathrm{Si}$ saturation is shown as red and undersaturation as blue whilst $\mathrm{O}$ rich initial conditions are squares and $\mathrm{O}$ poor conditions are circles. Numbers within symbols give the $\mathrm{CMB}$ heat flow at $3.5 \mathrm{Ga}$ and where models fail to maintain positive $E_{j}$ prior to inner core nucleation, symbols are crossed out. Also shown are the models of Davies and Greenwood (2022) (who examine $\mathrm{MgO}$ precipitation) where up, right and down triangles have a ppt. rate of $0,0.3$ and $1.5 \times 10^{-5} \mathrm{~K}^{-1}$ respectively and colours denote the core properties in terms of the density jump at the ICB (0.6 (light grey) and $1.0 \mathrm{~g} \mathrm{~cm}^{-3}$ (dark grey)) which represent bounding extremes of the density jump.

Fig. 8 shows the final compositions from the successful models in Table 2. Both low and high initial oxygen concentrations are broadly consistent with previous predictions of compositions which satisfy outer core density (Badro et al., 2014; Komabayashi, 2020), although we do not find compositions consistent with the inner core density jump (Davies et al., 2015). Note that we do not calculate the outer core density from our final model com- 
526 positions. The compositional space found to be consistent with outer core ${ }_{527}$ density Badro et al. (2014) is approximately linear and does not encompass ${ }_{528}$ equal, low concentrations of $\mathrm{Si}$ and O (Fig. 8). This suggests that our com529 positions are too simple, however, adding light elements to the liquid can 530 tune the density of the liquid as well as the Si solubility. 


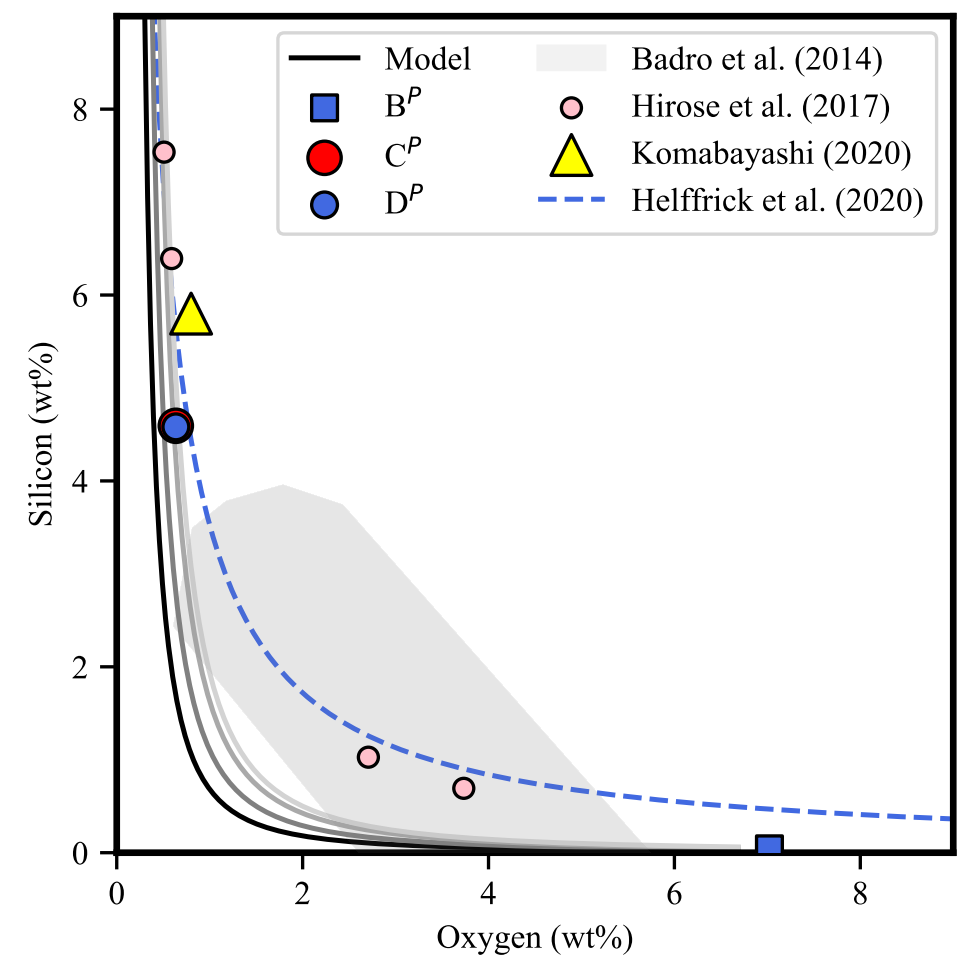

Figure 8: Final core Si and O compositions of successful thermal evolution models (red and blue symbols). The shaded region denotes the compositional space found to be consistent with outer core density from Badro et al. (2014) and the yellow triangle represents the same prediction from Komabayashi (2020). Predictions of current core composition at $4000 \mathrm{~K}$ from Hirose et al. (2017) (pink circles) and solubility from Helffrich et al. (2020) (blue dashed line) at the same temperature.

\section{Conclusion}

We find that the precipitation of Si allows the early core to cool more slowly than it would otherwise and supplies power to the geodynamo throughout Earth history. High $\mathrm{O}$ concentration in the core can reduce ancient core 
temperatures but requires an unreasonable mantle viscosity profile. Our more plausible low $\mathrm{O}$ cases result in higher CMB temperatures. The rheological transition of the magma ocean should occur between $40 \%$ to $60 \%$ melt fraction (Abe, 1997; Solomatov, 2015), rather than at the intersect of the liquidus or solidus, which would correspond to the occurrence of complete freezing and first partial melt, respectively. Our thermal histories for the low oxygen contents in particular suggest a long lived basal magma ocean. Although we do not include a magma ocean in our parameterisation of the mantle, this is more consistent with our chemical potentials and thermodynamic model as they both imply liquid-liquid interactions. For Si precipitation to power an early geodynamo the liquid core must have a low, perhaps less than 3 mol\%, O content, such that sufficient Si can be dissolved. In this scenario, transfer from the mantle may have increased the $\mathrm{O}$ content of the core as $\mathrm{Si}$ was removed.

\section{Author Contributions}

M.P. and D.A. conducted the ab initio portion of this project, producing chemical potentials. A.P. constructed the experimental database and S.G. developed the thermal evolution model codes. This project was the inception of C.D. who provided guidance alongside A.M.W.. A.J.W. conducted analysis of experimental data, constructed the thermodynamic model, conducted thermal evolution simulations and was primarily responsible for the writing of this manuscript, to which all authors contributed. 


\section{Acknowledgements}

We acknowledge the Natural Environment Research Council (NERC) Grant No. NE/T000228/1, which supports A.J.W., C.D., M.P., D.A and A.M.W.. M.P. and D.A. also receive support from NERC Grant No. NE/R000425/1. and S.G., A.P. and C.D. are supported by NSFGEO-NERC grant No. 1832462 (NSF) NE/T003855/1 (NERC).

Calculations were performed on the Monsoon2 system, a collaborative facility supplied under the Joint Weather and Climate Research Programme, a strategic partnership between the UK Met Office and NERC as well as on the U.K. national service Archer and the successing Archer2 service.

\section{Data Availability}

The dataset using in this study is available to download from the supplementary information. Details of the studies included are provided in the supplementary information.

\section{References}

Abe, Y., 1997. Thermal and chemical evolution of the terrestrial magma ocean. Physics of the Earth and Planetary Interiors 100, 27-39.

Alfè, D., Gillan, M., Price, G.D., 2002. Composition and temperature of the Earth's core constrained by combining ab initio calculations and seismic data. Earth and Planetary Science Letters 195, 91-98. 
Badro, J., Aubert, J., Hirose, K., Nomura, R., Blanchard, I., Borensztajn, S., Siebert, J., 2018. Magnesium partitioning between Earth's mantle and core and its potential to drive an early exsolution geodynamo. Geophysical Research Letters 45, 13-240.

Badro, J., Brodholt, J.P., Piet, H., Siebert, J., Ryerson, F.J., 2015. Core formation and core composition from coupled geochemical and geophysical constraints. Proceedings of the National Academy of Sciences 112, 1231012314 .

Badro, J., Côté, A.S., Brodholt, J.P., 2014. A seismologically consistent compositional model of Earth's core. Proceedings of the National Academy of Sciences 111, 7542-7545.

Badro, J., Siebert, J., Nimmo, F., 2016. An early geodynamo driven by exsolution of mantle components from Earth's core. Nature 536, 326-328.

Bouhifd, M.A., Jephcoat, A.P., 2003. The effect of pressure on partitioning of $\mathrm{Ni}$ and $\mathrm{Co}$ between silicate and iron-rich metal liquids: a diamond-anvil cell study. Earth and planetary science letters 209, 245-255.

Braginsky, S., 1963. Structure of the f layer and reasons for convection in the earth's core, in: Soviet Phys. Dokl., pp. 8-10.

Buffett, B.A., Huppert, H.E., Lister, J.R., Woods, A.W., 1996. On the thermal evolution of the Earth's core. Journal of Geophysical Research: Solid Earth 101, 7989-8006. 
Buffett, B.A., Seagle, C.T., 2010. Stratification of the top of the core due to chemical interactions with the mantle. Journal of Geophysical Research: Solid Earth 115.

Chidester, B.A., Rahman, Z., Righter, K., Campbell, A.J., 2017. Metalsilicate partitioning of $u$ : Implications for the heat budget of the core and evidence for reduced $\mathrm{u}$ in the mantle. Geochimica et Cosmochimica Acta 199, 1-12.

Davies, C., Greenwood, S., 2022. Thermo-chemical dynamics in Earth's core arising from interactions with the mantle. eartharxiv.org $0,0$.

Davies, C., Pozzo, M., Gubbins, D., Alfè, D., 2015. Constraints from material properties on the dynamics and evolution of Earth's core. Nature Geoscience 8, 678-685.

Davies, C.J., 2015. Cooling history of Earth's core with high thermal conductivity. Physics of the Earth and Planetary Interiors 247, 65-79.

Davies, C.J., Pozzo, M., Alfè, D., 2019. Assessing the inner core nucleation paradox with atomic-scale simulations. Earth and Planetary Science Letters $507,1-9$.

Davies, C.J., Pozzo, M., Gubbins, D., Alfè, D., 2020. Transfer of oxygen to Earth's core from a long-lived magma ocean. Earth and Planetary Science Letters 538, 116208. 
Driscoll, P., Bercovici, D., 2014. On the thermal and magnetic histories of Earth and Venus: Influences of melting, radioactivity, and conductivity. Physics of the Earth and Planetary Interiors 236, 36-51.

Du, Z., Boujibar, A., Driscoll, P., Fei, Y., 2019. Experimental constraints on an mgo exsolution-driven geodynamo. Geophysical Research Letters 46, 7379-7385.

Du, Z., Jackson, C., Bennett, N., Driscoll, P., Deng, J., Lee, K.K., Greenberg, E., Prakapenka, V.B., Fei, Y., 2017. Insufficient energy from MgO exsolution to power early geodynamo. Geophysical Research Letters 44, $11-376$.

Fischer, R.A., Campbell, A.J., Ciesla, F.J., 2017. Sensitivities of Earth's core and mantle compositions to accretion and differentiation processes. Earth and Planetary Science Letters 458, 252-262.

Fischer, R.A., Nakajima, Y., Campbell, A.J., Frost, D.J., Harries, D., Langenhorst, F., Miyajima, N., Pollok, K., Rubie, D.C., 2015. High pressure metal-silicate partitioning of $\mathrm{Ni}, \mathrm{Co}, \mathrm{V}, \mathrm{Cr}$, Si, and O. Geochimica et Cosmochimica Acta 167, 177-194.

Gomi, H., Ohta, K., Hirose, K., Labrosse, S., Caracas, R., Verstraete, M.J., Hernlund, J.W., 2013. The high conductivity of iron and thermal evolution of the Earth's core. Physics of the Earth and Planetary Interiors 224, 88103. 
Greenwood, S., Davies, C.J., Mound, J.E., 2021. On the evolution of thermally stratified layers at the top of Earth's core. Physics of the Earth and Planetary Interiors, 106763.

Gubbins, D., Alfè, D., Davies, C., Pozzo, M., 2015. On core convection and the geodynamo: Effects of high electrical and thermal conductivity. Physics of the Earth and Planetary Interiors 247, 56-64.

Gubbins, D., Alfe, D., Masters, G., Price, G.D., Gillan, M., 2004. Gross thermodynamics of two-component core convection. Geophysical Journal International 157, 1407-1414.

Helffrich, G., Hirose, K., Nomura, R., 2020. Thermodynamical modeling of liquid Fe-Si-Mg-O: Molten magnesium silicate release from the core. Geophysical Research Letters 47, e2020GL089218.

Hirose, K., Morard, G., Sinmyo, R., Umemoto, K., Hernlund, J., Helffrich, G., Labrosse, S., 2017. Crystallization of silicon dioxide and compositional evolution of the Earth's core. Nature 543, 99-102.

Hohenberg, P., Kohn, W., 1964. Inhomogeneous electron gas. Physical review $136, \mathrm{~B} 864$.

Holme, R., Olson, P., Schubert, G., 2015. Large-scale flow in the core. Treatise on geophysics 8, 107-130.

Jacobson, S.A., Rubie, D.C., Hernlund, J., Morbidelli, A., Nakajima, M., 
2017. Formation, stratification, and mixing of the cores of Earth and Venus. Earth and Planetary Science Letters 474, 375-386.

Jaupart, C., Labrosse, S., Lucazeau, F., Mareschal, J., 2007. 7.06temperatures, heat and energy in the mantle of the earth. Treatise on geophysics 7, 223-270.

Kohn, W., Sham, L.J., 1965. Self-consistent equations including exchange and correlation effects. Physical review 140, A1133.

de Koker, N., Steinle-Neumann, G., Vlček, V., 2012. Electrical resistivity and thermal conductivity of liquid Fe alloys at high $\mathrm{P}$ and $\mathrm{T}$, and heat flux in Earth's core. Proceedings of the National Academy of Sciences 109, 4070-4073.

Komabayashi, T., 2020. Thermodynamics of the system Fe-Si-O under high pressure and temperature and its implications for Earth's core. Physics and Chemistry of Minerals 47, 1-13.

Kresse, G., Furthmüller, J., 1996. Efficient iterative schemes for ab initio total-energy calculations using a plane-wave basis set. Physical review B $54,11169$.

Kresse, G., Joubert, D., 1999. From ultrasoft pseudopotentials to the projector augmented-wave method. Physical review b 59, 1758.

Labrosse, S., 2015. Thermal evolution of the core with a high thermal conductivity. Physics of the Earth and Planetary Interiors 247, 36-55. 
Labrosse, S., Poirier, J.P., Le Mouël, J.L., 2001. The age of the inner core. Earth and Planetary Science Letters 190, 111-123.

Landeau, M., Olson, P., Deguen, R., Hirsh, B.H., 2016. Core merging and stratification following giant impact. Nature Geoscience 9, 786-789.

Li, Y., Vočadlo, L., Alfè, D., Brodholt, J., 2019. Carbon partitioning between the Earth's inner and outer core. Journal of Geophysical Research: Solid Earth 124, 12812-12824.

Ma, Z., 2001. Thermodynamic description for concentrated metallic solutions using interaction parameters. Metallurgical and Materials Transactions B $32,87-103$.

Mittal, T., Knezek, N., Arveson, S.M., McGuire, C.P., Williams, C.D., Jones, T.D., Li, J., 2020. Precipitation of multiple light elements to power Earth's early dynamo. Earth and Planetary Science Letters 532, 116030.

Nimmo, F., 2015a. Energetics of the core, in: Schubert, G. (Ed.), Treatise on geophysics 2nd Edn. Elsevier, Amsterdam. volume 8, p. 27-55.

Nimmo, F., 2015b. Thermal and compositional evolution of the core. Treatise on Geophysics 9, 201-219.

Nosé, S., 1984. A unified formulation of the constant temperature molecular dynamics methods. The Journal of chemical physics 81, 511-519. 
O’Rourke, J.G., Stevenson, D.J., 2016. Powering Earth's dynamo with magnesium precipitation from the core. Nature 529, 387-389.

Perdew, J.P., Chevary, J.A., Vosko, S.H., Jackson, K.A., Pederson, M.R., Singh, D.J., Fiolhais, C., 1992. Atoms, molecules, solids, and surfaces: Applications of the generalized gradient approximation for exchange and correlation. Physical review B 46, 6671.

Pozzo, M., Davies, C., Gubbins, D., Alfè, D., 2012. Thermal and electrical conductivity of iron at Earth's core conditions. Nature 485, 355-358.

Pozzo, M., Davies, C., Gubbins, D., Alfe, D., 2019. FeO content of Earth's liquid core. Physical Review X 9, 041018.

Rubie, D.C., Jacobson, S.A., Morbidelli, A., O’Brien, D.P., Young, E.D., de Vries, J., Nimmo, F., Palme, H., Frost, D.J., 2015. Accretion and differentiation of the terrestrial planets with implications for the compositions of early-formed solar system bodies and accretion of water. Icarus 248, 89-108.

Rudolph, M.L., Lekić, V., Lithgow-Bertelloni, C., 2015. Viscosity jump in earth's mid-mantle. Science 350, 1349-1352.

Solomatov, V., 2015. Magma oceans and primordial mantle differentiation, in: Schubert, G. (Ed.), Treatise on geophysics. Elsevier, Amsterdam. volume 10, pp. 81-104. 
Suer, T.A., Siebert, J., Remusat, L., Menguy, N., Fiquet, G., 2017. A sulfurpoor terrestrial core inferred from metal-silicate partitioning experiments. Earth and Planetary Science Letters 469, 84-97.

Takafuji, N., Hirose, K., Mitome, M., Bando, Y., 2005. Solubilities of o and si in liquid iron in equilibrium with $(\mathrm{mg}, \mathrm{fe})$ sio3 perovskite and the light elements in the core. Geophysical Research Letters 32.

Tarduno, J.A., Cottrell, R.D., Watkeys, M.K., Hofmann, A., Doubrovine, P.V., Mamajek, E.E., Liu, D., Sibeck, D.G., Neukirch, L.P., Usui, Y., 2010. Geodynamo, solar wind, and magnetopause 3.4 to 3.45 billion years ago. science $327,1238-1240$.

Usui, Y., Tsuchiya, T., 2010. Ab initio two-phase molecular dynamics on the melting curve of $\mathrm{SiO}_{2}$. Journal of Earth Science 21, 801-810.

Zhang, Y., Hou, M., Liu, G., Zhang, C., Prakapenka, V.B., Greenberg, E., Fei, Y., Cohen, R., Lin, J.F., 2020. Reconciliation of experiments and theory on transport properties of iron and the geodynamo. Physical review letters 125, 078501.

Zhang, Y., Luo, K., Hou, M., Driscoll, P., Salke, N.P., Minár, J., Prakapenka, V.B., Greenberg, E., Hemley, R.J., Cohen, R., et al., 2022. Thermal conductivity of fe-si alloys and thermal stratification in earth's core. Proceedings of the National Academy of Sciences 119. 